\title{
FDG PET/CT in Malignant Transformation of Peripheral Nerve Sheath Tumor in Known Case NF-1: A Case Report
}

Sibu Jacob B, Kiran Kumar JK*, Alok Dixit, Nirav Thaker R and Inder Talwar

Department of Nuclear Medicine and PET -CT, Bombay Hospital and Medical Research Centre, Mumbai, India

\begin{abstract}
Neurofibromatosis type 1 is an autosomal dominant disease. The worldwide estimated prevalence is 1 in 3000. Germline mutation in the NF1 tumor suppressor gene on long arm of chromosome 17 appears to be the causative factor. Patients with plexiform neurofibromas shows higher risk of transformation into malignant pathology also referred to as malignant peripheral nerve sheath tumors (MPNST). The relative lifetime risk is expected to be around $8-13 \%$. Histology remains gold standard for diagnosing MPNST, but requires complete excision which is technically difficult. As a combined metabolic-anatomic method, PET/CT using the radiotracer 18F-FDG allows different parameters of tumours to be analysed simultaneously. It also helps to avoid unnecessary surgeries in benign cases. Hybrid PET/MRI will play a crucial role in future for evaluating nerve sheath tumours in NF1 patients by combining the pros of both the modalities.
\end{abstract}

\section{Introduction}

Neurofibromatosis type 1 (NF1, a.k.a. von Recklinghausen's disease) is an autosomal dominant disease. The worldwide estimated prevalence is 1 in 3000 [1]. Germline mutation in the NF1 tumor suppressor gene on long arm of chromosome 17 (gene locus q11.2) appears to be the causative factor. It codes for the cytoplasmic protein - neurofibromin [2,3]. Patients with NF-1 typically manifest as caféau-lait spots, cutaneous and plexiform neurofibromas (BNF), optic glioma, Lisch nodules and bone dysplasia [4]. While several clinical features of NF1 are already seen at birth, few other develop later in the course of the disease, with number of cutaneous neurofibromas increasing with age [5]. Patients with plexiform neurofibromas shows higher risk of transformation into malignant pathology also referred to as Malignant Peripheral Nerve Sheath Tumors (MPNST). The relative lifetime risk is expected to be around $8-13 \%$ [6,7]. MPNST are highly malignant and aggressively metastatic which shows poor prognosis. Early resection appears to be the only curative option. Hence early detection of these malignant transformations is a must.

Both benign and malignant lesions, especially when present together in a patient, have similar clinical manifestations such as changes in consistency or size, unremitting pain, or new neurological findings [8]. Histology remains gold standard for diagnosing MPNST, but requires complete excision which is technically difficult. And if core biopsy is performed, the focus of malignant change, especially in large heterogeneous tumour, may be missed. Morphologic imaging cannot reliably differentiate malignant transformed lesions from benign ones, especially in tumours with significant heterogeneity [9]. Patients with NF1, when evaluated with PET, has demonstrated overall good sensitivity $(89-100 \%)$ and specificity $(72-95 \%)$ in differentiating BNF from MPNSTs [10-12]. As a combined metabolic-anatomic method, $\mathrm{PET} / \mathrm{CT}$ using the radiotracer $18 \mathrm{~F}-\mathrm{FDG}$ allows different parameters of tumours to be analyzed simultaneously. FDG-PET/CT metabolic imaging has been shown to be able to detect these malignant pathology, with positive correlation between FDG avidity and histologic grade [13].

Here we discuss the utility of FDG PET/CT in one such case which influenced the prognosis and treatment strategy.

\section{Case Report}

A 35 year old male, a known case of NF-1, diagnosed in 2016 presented with progressive left foot weakness, left lower limb pain and walking difficulty since 4 months. MRI of thigh done on February 17 showed plexiform neurofibroma along left sciatica nerve. He was then referred to Nuclear medicine department for further evaluation. FDG PET with contrast enhanced CT was performed which demonstrated intense FDG avid (SUV max 20.3) heterogeneously enhancing large lobulated soft tissue mass measuring $32.8 \times 7.8 \times 7.4 \mathrm{~cm}$ along left sciatica nerve in mid and distal thigh extending minimally along proximal peroneal nerve (Figure 1). There were multiple other non FDG avid hypodense lesion along nerve roots of entire spine, carotid space, bilateral axillary and thigh. Multiple non FDG avid bilateral pulmonary and subcutaneous nodules were also seen. Histopathological examination done from the FDG avid mass revealed spindle cells arranged in sweeping fascicles, also cells with a malignant rhabdoid phenotype and tumour giant cells in a diffuse pattern. Mitotic activity is brisk and focal necrosis is noted. These cells expressed S-100 protein and CD34 and negative for desmin, SMA and myogenin. These features suggested MPNST.

He then underwent excision surgery (May 2017), 3 cycles chemotherapy (till August 2017). PET/CT done on August 2017 suggested no residual lesion in the operated site with mild increase in FDG avidity and size of pulmonary nodules (Figure 2). He then underwent radiotherapy (till November 2017). After 3 months he presented with chest pain and left pleural effusion. Cytology of the pleural fluid was positive for malignant cells. FDG PET with contrast enhanced CT done on February 2018 (Figure 3) revealed interval new FDG avid pulmonary and pleural metastases with moderate bilateral

*Corresponding author: Kiran Kumar JK, Consultant and Head, Department of Nuclear Medicine and PET -CT, Bombay Hospital and Medical Research Centre Mumbai, India, Tel: 9872995758; E-mail: jkkirannayak@gmail.com

Received February 28, 2018; Accepted March 02, 2018; Published March 05 2018

Citation: Sibu Jacob B, Kiran Kumar JK, Dixit A, Nirav Thaker R, Talwar I (2018) FDG PET/CT in Malignant Transformation of Peripheral Nerve Sheath Tumor in Known Case NF-1: A Case Report. J Nucl Med Radiat Ther 9: 356. doi: 10.4172/2155-9619.1000356

Copyright: (c) 2018 Sibu Jacob B, et al. This is an open-access article distributed under the terms of the Creative Commons Attribution License, which permits unrestricted use, distribution, and reproduction in any medium, provided the original author and source are credited. 

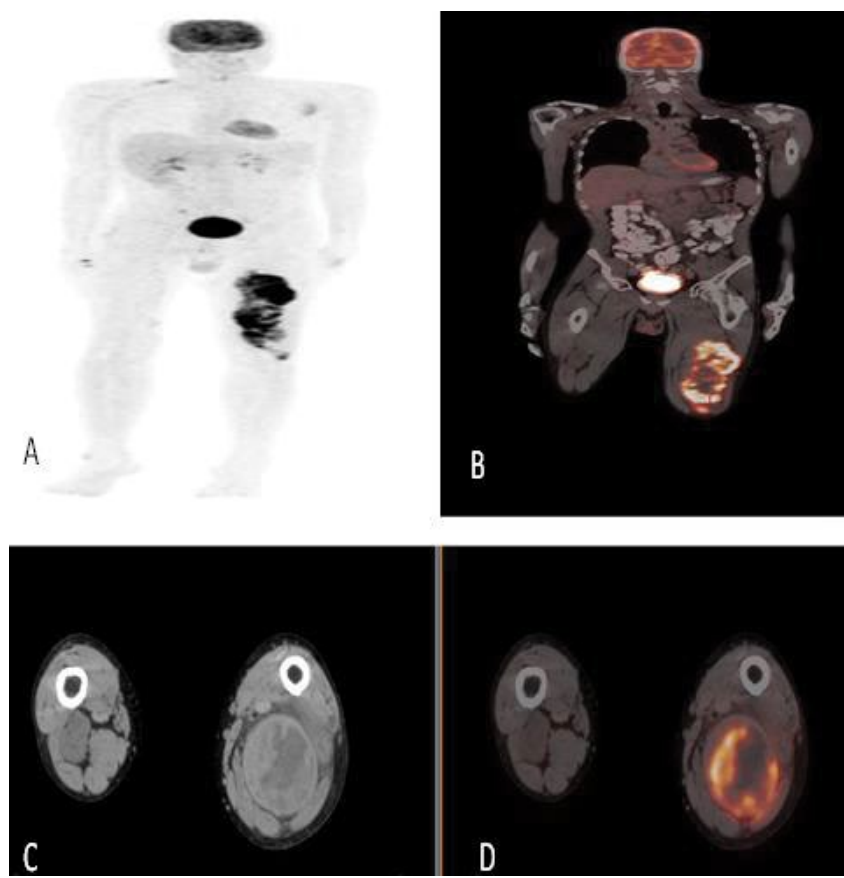

Figure 1: (A) MIP (maximum intensity projection) shows intense FDG avid (SUVmax 20.3) lobulated soft tissue mass in the region of mid and distal thigh (B) Coronal FDG PET and CT fused images shows central necrotic areas within hypermetabolic soft tissue lesion (along the course of sciatic nerve) (C,D) Axial CT and PET/CT fused images at level of left midthigh shows targe sign (central hypodense areas and peripheral enhancing lesion) on CT with peripherally hypermetabolic and centrally photopenic area on PET. On biopsy it was MPNST.

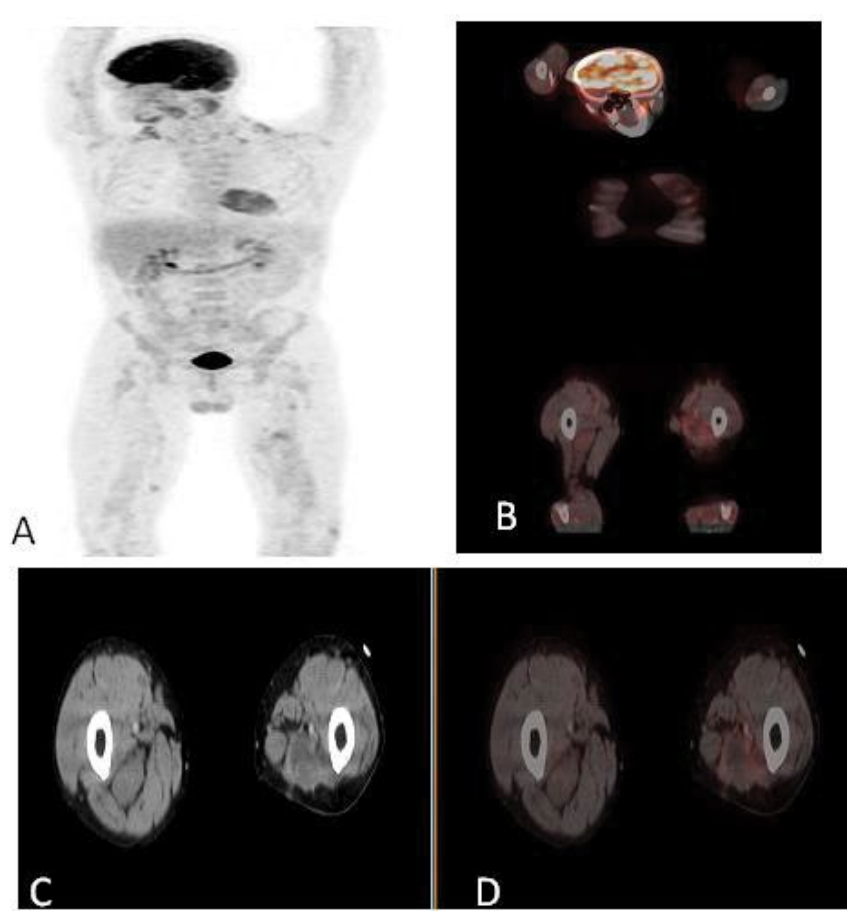

Figure 2: (A) MIP (Maximum Intensity Projection) shows mild FDG uptake along the left mid and distal thigh; $(B)$ Coronal $(C, D)$ Axial FDG PET and CT fused images shows post-operative changes with no residual / recurrent changes.
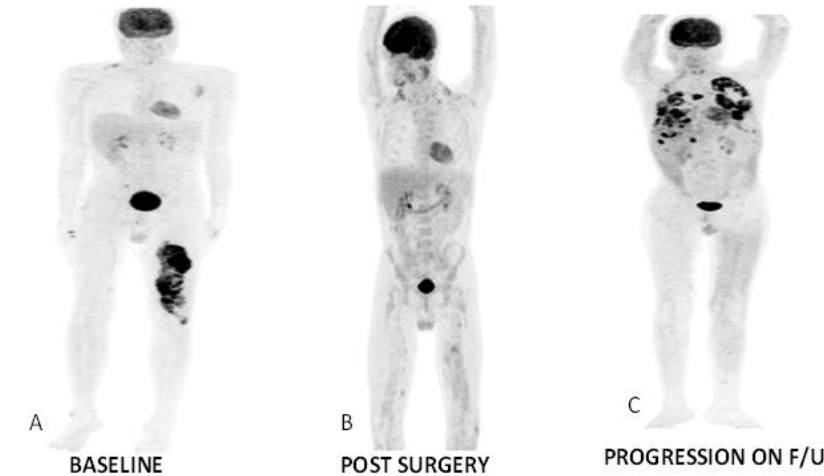

Figure 3: MIP (Maximum Intensity Projection) images: A: (Baseline) Intense FDG uptake along the left mid and distal thigh; B: (Post surgery and chemotherapy) MIP shows no abnormal FDG uptake along the left mid and distal thigh; C, (Follow up) MIP shows no abnormal FDG uptake along the left mid and distal thigh, however there was FDG avid pulmonary metastasis.

pleural effusion and unchanged previously seen neurofibroma lesions. However there was no recurrent/residual lesion in the operated site.

\section{Discussion}

MPNST's (a.k.a. malignant schwannoma, neurosarcoma) is a life threatening disease, prone to transformation from previous benign neurofibroma in patients with NF-1. The classical features seen in MPNST significantly overlaps with benign neurofibroma. MR and CT also cannot reliably differentiate benign from malignant lesion. Histological assessment mostly is either technically difficult or gives false negative results.

Distinguishing features in MR for malignant transformation included largest dimension of mass, heterogenicity of lesion, peripheral enhancement pattern, perilesional edema and intratumoral cystic lesion. The presence of 2 or more suggested malignancy with sensitivity of $61 \%$ and specificity of $90 \%$ in a study conducted by Junjiwasa, et al. [9]. On CT scan it appears hypodense due to the presence of lipid rich Schwann cells, adipocytes, accumulated interstitial fluid and cystic areas resulting from infarctions and necrosis, particularly in cases of larger and malignant nerve sheath tumors. In addition, perineural fat tissue can be involved with the growth of diffuse plexiform neurofibromas, thereby causing lower density values on CT [14]. Contrast CT study often reveals heterogenous enhancement due to differences in cellularity and collagen density and also due to cystic areas [15]. Hence morphologic imaging cannot reliably differentiate benign from malignant transformed lesions, though it can be used to determine site and extent of tumour.

PET on the other hand detects MPNST with high specificity and sensitivity [16], and also permits whole-body surveillance. Simultaneous CT is aids in anatomic correlation and is valuable in cases with typical pulmonary and osseous metastases. There are three approaches to interpret these studies:

1. Visual assessment: It is fast but operator-dependent and with low reproducibility.

2. Quantitative assessment: Standardized uptake value (SUV cutoff)with sensitivity, specificity, positive predictive value and negative predictive value for separating MPNSTs from BNFs of $91 \%, 84 \%, 67 \%$, and $96 \%$ versus $91 \%, 81 \%, 63 \%$, and $96 \%$, 
respectively, on 4-hour delayed imaging, and showed that the mean SUVmax was significantly higher for MPNSTs than BNFs on both early scans $(6.5$ vs. $2.0, \mathrm{P}<0.01)$ and delayed imaging $(8.3$ vs. $2.3, \mathrm{P}<0.02)$ [17]. However the quantitative assessment is variable but usually correlates with the aggressiveness of the disease.

3. SUVmax of tumour to SUV mean of background (liver) ratio [T/L ratio]: It is more reproducible with less variability with NPV and PPV of $98.8 \%$ and $65.1 \%$ respectively. The recommended $\mathrm{T} / \mathrm{L}$ ratio is $<1.5$ for monitoring and $>1.5$ to intervene (surgery/ biopsy) [18].

Serial follow up with PET and MRI can be done for evaluating abnormal growth pattern, interval increase in FDG uptake and MRI features suggestive of malignant transformation. In NF1 patients, a negative PET excludes a malignant transformation with high likelihood, the NPV of being as high as $100 \%$ in newer studies [19]. Characteristically, benign tumours show low grade FDG concentration and due to low cellularity and presence of lipid laden Schwann cells [20]. In addition, the metabolic activity (SUVmax) / FDG avidity is a better predictor for overall survival than histological grading [21].

In our case, the patient with NF-1 who was initially diagnosed with multiple well defined benign nerve sheath tumour throughout the body, later on had symptoms of progressive left foot weakness, left lower limb pain and walking difficulty and was diagnosed with malignant transformation on FDG PET/CT which was histologically proven. This finding suggested the grave prognosis of the disease which unfortunately progressed as pulmonary and pleural metastases.

The potential role of FDG PET is also studied in guiding biopsy, preoperative staging and treatment decision. It reduces the number of unwanted surgeries for benign cases. In some institutions, lesions with low SUV values were suggested clinical or radiological follow up; those with intermediate values went for biopsy or re-evaluation and those with high values or suspected to be malignant could undergo guided biopsy or resection.

\section{Conclusions}

18F-FDG-PET/CT allows highly sensitive and specific detection of MPNST in patients with NF1 and should be used when a malignant transformation of a nerve sheath tumour is suspected, particularly for staging. It also helps to avoid unnecessary surgeries in benign cases. Hybrid PET/MRI will play a crucial role in future for evaluating nerve sheath tumours in NF1 patients by combining the pros of both the modalities.

\section{References}

1. Friedman JM (1999) Epidemiology of neurofibromatosis type 1. Am J Med Genet 89: 1-6.

2. Williams VC, Lucas J, Babcock MA (2009) Neurofibromatosis type 1 revisited Pediatrics 123: 124-133.

3. Hirbe AC, Gutmann DH (2014) Neurofibromatosis type 1: A multidisciplinary approach to care. Lancet Neurol 13: 834-843.
4. Ferner RE, Huson SM, Thomas N (2007) Guidelines for the diagnosis and management of individuals with neurofibromatosis 1. J Med Genet 44: 81-88.

5. Duong TA, Bastuji-Garin S, Valeyrie-Allanore L (2011) Evolving pattern with age of cutaneous signs in neurofibromatosis type 1: A cross-sectional study of 728 patients. Dermatology 222: 269-273.

6. Nguyen R, Jett K, Harris GJ, Cai W, Friedman JM, et al. (2014) Benign whole body tumor volume is a risk factor for malignant peripheral nerve sheath tumors in neurofibromatosis type 1. J Neurooncol 116: 307-313.

7. Evans DG, Baser ME, McGaughran J, Sharif S, HowardE, et al. (2002) Malignant peripheral nerve sheath tumours in neurofibromatosis $1 . \mathrm{J}$ Med Genet 39: 311-314.

8. Ferner RE, Gutmann DH (2002) International consensus statement on malignant peripheral nerve sheath tumours in neurofibromatosis 1. Cancer Res 62: 1573-1577.

9. Wasa J, Nishida Y, Tsukushi S (2010) MRI features in the differentiation of malignant peripheral nerve sheath tumors and neurofibromas. Am J Roentgenol 194: 1568-1574

10. Fisher MJ, Basu S, Dombi E (2008) The role of [18F]-fluorodeoxyglucose positron emission tomography in predicting plexiformneurofibroma progression. J Neurooncol 87: 165-171.

11. Karabatsou K, Kiehl TR, Wilson DM, Hendler A, Guha A (2009) Potential role of 18fluorodeoxyglucose positron emission tomography/computed tomography in differentiating benign neurofibroma from malignant peripheral nerve sheath tumor associated with neurofibromatosis 1. Neurosurgery 65: A160-A170.

12. Moharir M, London K, Howman-Giles R, North K (2010) Utility of positron emission tomography for tumour surveillance in children with neurofibromatosis type 1. Eur J Nucl Med Mol Imaging 37: 1309-1317.

13. Benz MR, Tchekmedyian N, Eilber FC, Federman N, Czernin J, et al. (2009) Utilization of positron emission tomography in the management of patients with sarcoma. Curr Opin Oncol 21: 345-351.

14. Kumar AJ, Kuhajda FP, Martinez CR (1983) Computed tomography of extracranial nerve sheath tumors with pathological correlation. J Comput Assist Tomogr 7: 857-865.

15. Cohen LM, Schwartz AM, Rockoff SD (1986) Benign schwannomas: pathologic basis for CT inhomogeneities. Am J Roentgenol 147: 141-143.

16. Warbey VS, Ferner RE, Dunn JT (2009) [18F]FDG PET/CT in the diagnosis of malignant peripheral nerve sheath tumours in neurofibromatosis type-1. Eur $J$ Nucl Med Mol Imaging 36: 751-757.

17. Chirindel A, Chaudhry M, Blakeley JO, Wahl R (2015) 18FFDG PET/CT qualitative and quantitative evaluation in neurofibromatosis type 1 patients for detection of malignant transformation: Comparison of early to delayed imaging with and without liver activity normalization. J Nucl Med 56: 379-385.

18. Combemale P, Allanore VL, Giammarile F, Pinson S, Guillot B (2014) Utility of 18FFDG PET with a semiquantitative index in the detection of sarcomatous transformation in patients with neurofibromatosis type 1. PLoS ONE 9: e85954.

19. Salamon J, Derlin T, Bannas P (2013) Evaluation of intratumoural heterogeneity on ${ }^{18} \mathrm{~F}$-FDG PET/CT for characterization of peripheral nerve sheath tumours in neurofibromatosis type 1. Eur J Nucl Med Mol Imaging 40: 685-692.

20. Cardona S, Schwarzbach M, Hinz U, Dimitrakopoulou-Strauss A, Attigah N et al. (2003) Evaluation of F18-deoxyglucose positron emission tomography (FDG-PET) to assess the nature of neurogenic tumours. Eur J Surg Oncol 29 536-541.

21. Brenner W, Friedrich RE, Gawad KA (2006) Prognostic relevance of FDG $\mathrm{PET}$ in patients with neurofibromatosis type-1 and malignant peripheral nerve sheath tumours. Eur J Nucl Med Mol Imaging 33: 428-432. 